\title{
RELAÇÃO DA TEMPERATURA DA SOLUÇÃO DE DIÁLISE E A HIPOTENSÃO ARTERIAL SINTOMÁTICA OBSERVADA DURANTE SESSÕES DE HEMODIÁLISE EM PACIENTES COM INSUFICIÊNCIA RENAL CRÔNICA*
}

\author{
RELATIONSHIP BETWEEN DIALYSIS SOLUTION TEMPERATURE AND \\ SYMPTOMATIC LOW BLOOD PRESSURE DURING HEMODIALYSIS FOR END \\ STAGE RENAL DISEASE PATIENTS
}

\begin{abstract}
RELACIÓN DE LA TEMPERATURA DE LA SOLUTION DE DIÁLISIS Y LA HIPOTENSIÓN ARTERIAL SINTOMÁTICA OBSERVADA DURANTE SESIONES DE HEMODIALISIS EN PACIENTES CON INSUFICIENCIA RENAL CRÓNICA
\end{abstract}

\author{
Clélia Beltrame Soares ** \\ Emilia Yaeko Ochiro *** \\ Natalina Toyoko Sannomiya ***
}

Soares CB, Ochiro EY, Sannomiya NT. Relação da temperatura da solução de diálise e a hipotensão arterial sintomática observada durante sessões de hemodiálise em pacientes com insuficiência renal crônica. Rev Esc Enferm USP 2001; 35(4): 346-53.

\section{RESUMO}

Realizado estudo prospectivo em um grupo de 21 pacientes portadores de insuficiência renal crônica que apresentavam hipotensão arterial no decorrer da hemodiálise. Avaliada a pressão arterial durante duas sessões com dialisato a $35{ }^{9} \mathrm{C}$ e duas a $37^{\circ} \mathrm{C}$, observou-se que as pressões sistólica e diastólica, nas temperaturas estudadas, mostraram diferenças estatisticamente significativas quando comparadas aos valores iniciais pré-diálise, queda progressiva das pressões com prevalência de episódios hipotensivos na 35 e 45 horas de tratamento em ambas temperaturas, diminuição de 7,69\% das hipotensões com dialisato a $35^{\circ} \mathrm{C}$ e importante queixa de sensação de frio, tornando o tratamento desconfortável.

PALAVRAS-CHAVE: Diálise. Hipotensão. Hemodiálise. Insuficiência renal crônica.

\begin{abstract}
This prospective study was designed to evaluate hypotension in a group of 21 end stage renal disease patients (ESRD) on hemodialysis, with syntomatic low blood pressure. The arterial blood pressure was recorded at 4 consecutive dialysis sessions, two at $35^{\circ} \mathrm{C}$ and two at $37^{\circ} \mathrm{C}$ dialysate temperature. In both situations, systolic and diastolic pressures, measured during dialysis, were different from the pre-dialysis value, with progressive lowering up to the end of the procedure. Cold dialysis did not protect from lowering the blood pressure in an amount similar to that occuring at $37^{\circ} \mathrm{C}$.
\end{abstract}

KEYWORDS: Dialysis. Hypotension. Hemodialysis. Kidney failure chronic.

\section{RESUMEN}

Realizado estudio prospectivo en un grupo de 21 pacientes portadores de insuficiencia renal crónica que presentaban hipotensión arterial en el decorrer de la hemodiálisis. Evaluada la presión arterial durante dos sesiones con dialisato a $35^{\circ} \mathrm{C}$ y dos a $37^{\circ} \mathrm{C}$ se observó que las presiones sistolica y diastólica, en las temperaturas estudiadas, mostraron diferencias estadisticamente significativas cuando comparadas a los valores iniciales predialisis, caida progresiva de las presiones con prevalencia de episodios hipotensivos en la $3^{9}$ y $4^{5}$ horas de tratamiento en ambas temperaturas. Disminuición de $7,69 \%$ de las hipotensiones con dialisato a $35^{\circ} \mathrm{C}$ e importante queja de sensación de frio, tornando el tratamiento desconfortable.

PALABRAS-ClAve: Diálisis. Diálisis fria. Hipotensión arterial. Hemodiálisis.

\footnotetext{
* Trabalho desenvolvido para obtenção de título de Especialista em Enfermagem em Nefrologia pela Pontifícia Universidade Católica do Paraná em 1999, orientado pelo Prof. Dr. Altair J. Mocelin.

** Enfermeira do Hospital Universitário Regional do Norte do Paraná E-mail; bsoares@sercomtel.com.br

*** Enfermeiras do Instituto do Rim de Londrina.
} 


\section{INTRODUÇÃO}

A insuficiência renal crônica (IRC) resulta de uma progressiva perda de néfrons, que são as unidades funcionais dos rins; durante esse processo os néfrons remanescentes sofrem alterações para adaptação nas suas funções secretoras, reabsortivas e metabólicas, com a finalidade de manter um relativo equilíbrio no meio interno do organismo. Apesar dessa tentativa, ocorre o que se chama de azotemia, que são alterações bioquímicas nos niveis sangüineos de uréia, creatinina e outras substâncias nitrogenadas que normalmente são excretadas através da urina e estão retidas na insuficiência renal. O termo uremia descreve as alterações clínicas resultantes dessa doença (1).

Uma vez que a doença renal tenha sido estabelecida e a sua forma crônica se desenvolve, o padrão de deterioração evolui inexoravelmente para a fase terminal, ou seja, a necessidade de terapia substitutiva da função renal (2-3).

As opções terapêuticas para o tratamento dos pacientes em fase terminal de doença renal são a hemodiálise (HD), diálise peritoneal intermitente (DPI), diálise peritoneal ambulatorial contínua (DPAC), diálise peritoneal cíclica contínua (DPCC) e suas variações e o transplante renal, seja de doador vivo ou cadavérico ${ }^{(2,4)}$.

\section{Hemodiálise}

A hemodiálise consiste em uma técnica de filtração do sangue utilizando dois processos fisicos: difusão e ultrafiltração. O transporte por difusão é o mecanismo primário para remoção de toxinas pela hemodiálise. Esse processo ocorre quando um gradiente de concentração de um soluto é estabelecido através de uma membrana semipermeável; então, o soluto se difunde pela membrana do lado mais concentrado para o lado menos concentrado. A taxa de difusão de transporte através dessa membrana aumenta com o aumento na diferença de concentração, um aumento na superficie de troca da membrana e um aumento no coeficiente de transferência de massa da membrana. O outro processo é a ultrafiltração (UF), no qual a remoção de fluidos é feita por meio da aplicação de um gradiente de pressão hidrostática ou osmótica através da membrana; dessa forma, o fluido atravessa a membrana do local de maior pressão para o de menor ${ }^{(5-7)}$.

O hemodialisador é um filtro com uma membrana semipermeável que possui uma grande área de superficie de troca sendo o sangue perfundido por um lado e o fluido de diálise pelo outro. Solutos em que a concentração é maior no sangue do que no fluido de diálise se difundirão através de gradiente de concentração, do sangue para o dialisato (5).

Apesar da hemodiálise fornecer uma dramática reversão da toxicidade urêmica, não se deve deixar de considerar que ela, na sua melhor eficiência é apenas uma aproximação da função renal natural. Ela consegue remover algumas toxinas urêmicas e fornecer uma correção adequada para as alterações ácidobásicas e hidroeletrolíticas do organismo, porém, não restaura qualquer função endócrina ou metabólica como os rins normais (5).

A hemodiálise é responsável, diretamente, pela manutenção da vida de mais de 100.000 pessoas nos EUA. Vários anos foram adicionados à vida dessas pessoas que, sem esta importante intervenção médica, teriam morrido pela IRC (8).

A prevalência no Brasil, em 1996, era de 166 pacientes em tratamento dialítico por milhão de pessoas. A incidência é de 70 novos pacientes por ano em tratamento por milhão de habitantes. Nos Estados Unidos a incidência em 1992 foi de 214 pacientes por milhão e a prevalência de 791 pacientes por milhão. As principais causas nos Estado Unidos que levam à insuficiência renal crônica e conseqüente tratamento hemodialítico é o diabetes mellitus $(36,2 \%)$ seguida de hipertensão arterial $(30,1 \%)$ e glomerulonefrites $(12,9 \%)$. No Brasil é indicado como principal causa a glomerulonefrite crônica $(23 \%)$, seguida de nefroesclerose hipertensiva $(22 \%)$ e diabetes mellitus $(17 \%){ }^{(9)}$.

A hemodiálise é uma terapia segura, com risco estimado de uma morte para cada 75.000 tratamentos nos EUA. Além da discussão sobre as complicações dessa modalidade terapêutica, devem se considerar para a morbidade e mortalidade desses individuos em tratamento hemodialítico as condições clínicas básicas do paciente, como idade, diabetes mellitus, insuficiência coronariana, insuficiência cardiaca, doença hepática, desnutrição, entre outros ${ }^{(8)}$.

Embora os beneficios da hemodiálise sejam inquestionáveis, muitas complicações podem ocorrer durante esse procedimento. As complicações mais comuns ordenadas crescentemente, são febre e calafrios $(<1 \%)$, prurido $(5 \%)$, dor lombar $(2-5 \%)$, dor torácica ( $2-5 \%)$, cefaléia $(5 \%)$, náuseas e vômitos $(5-15 \%)$, cãibras (5-20\%) e a hipotensão arterial (20-30\% das diálises). Entre as complicações menos comuns, porém mais sérias, estão a síndrome do desequilíbrio, reações de hipersensibilidade, arritmia cardíaca, tamponamento cardíaco, hemorragia intracraniana, convulsão, hemólise e embolia gasosa( ${ }^{(10)}$.

A hipotensão arterial sintomática observada durante a hemodiálise é a causa mais freqüente de morbidade em pacientes com IRC, nessa forma de tratamento. Esta complicação ocorre em cerca de 20 a $30 \%$ dos procedimentos realizados e é mais comum em mulheres e em idosos. A hipotensão arterial sintomática é definida como pressão arterial sistólica abaixo de 90 mmHg ou queda em $25 \%$ desse valor quando o paciente apresentar uma pressão sistólica basal de 90-100 mmHg e associados a sintomas como zumbido, bocejos, fraqueza muscular, náuseas e vômitos, cãibras, sudorese, taquicardia, dor precordial e confusão ment(15-11).

A etiologia da hipotensão arterial durante o procedimento hemodialítico é multifatorial, podendo estar relacionada com a baixa reserva cardíaca, neuropatia autonômica, declínio da osmolalidade e do 
volume plasmático (12,14), arritmia cardíaca, hipocalemia (13), uso de solução de diálise de acetato de sódio, alta temperatura do dialisato, biocompatibilidade da membrana de diálise e alteração nos reflexos circulatórios (16).

A disfunção sistólica do ventrículo esquerdo é o principal fator de risco para os episódios de hipotensão, além de uma redução na complacência do sistema venoso. De um modo geral, a severidade da hipotensão intradialítica varia de acordo com a condição cardiovascular basal e o volume de fluido removido (17).

Existem dois tipos de hipotensão arterial relacionada à diálise; um tipo episódico que ocorre tipicamente ao final do tratamento e um segundo, que é uma forma crônica e persistente, que ocorre em um grupo de pacientes que tem pressão sangüinea sistólica pré-diálise menor que 90-100 $\mathrm{mmHg}$ e que freqüentemente diminui mais durante a diálise (18)

As causas desse segundo tipo de hipotensão tem sido relacionadas a uma disfunção autonômica ou a uma diminuição da reatividade de vasoconstritores como a angiotensina II, vasopressina, noradrenalina e pelo aumento do óxido nítrico, um importante regulador do tônus vascular (18).

O primeiro tipo de hipotensão, que surge após transcorrido metade do tempo da diálise, pode estar relacionado à decréscimos excessivos e rápidos no volume sangüíneo resultando em uma diminuição do débito cardíaco e conseqüente instalação desta, uma vez que para se manter o volume sangüineo durante a hemodiálise é necessário um reenchimento rápido do compartimento sangüíneo à partir dos espaços circunvizinhos. O ideal para evitar a hipotensão relacionada a esta causa seria a utilização de máquinas com dispositivo de controle de ultrafiltração; que o peso interdialítico não excedesse a 1 kg/dia; que não ocorresse ultrafiltração excessiva, ou seja, inferior ao "peso seco" do paciente e que a solução de diálise contivesse uma concentração de sódio igual ou superior ao plasmático $(10,14,18)$

Alguns pacientes experimentam hipotensão arterial em menos de uma hora de início da sessão de hemodiálise, havendo importante redução no débito cardíaco e na resistência vascular periférica, com volemia inalterada. Isto sugere que a redução no fluxo circulatório não se faz pela diminuição no volume sanguíneo, mas pela redução no retorno venoso ou por disfunção cardiaca ou ambos (19)

Durante a ultrafiltração isolada, na ausência de qualquer fluido de diálise, um progressivo aumento na resistência vascular sistêmica total mantém a pressão arterial enquanto o plasma é removido. Quando o processo de difusão é adicionado à ultrafiltração, ou seja, a terapia hemodialitica convencional, a vasoconstrição é menos eficiente durante o processo de remoção de líquido. Além disso, o débito cardíaco e a pressão arterial são mantidos pelo aumento na freqüência cardíaca e, em algumas vezes, pelo aumento na contratilidade miocárdica(8). Durante o processo de ultrafiltração, o volume de fluido plasmático é removido do espaço intravascular e o aumento da pressão osmótica plasmática provoca um movimento do líquido intersticial e intracelular para repor o volume plasmático. A hipotensão surge quando a taxa de retirada do volume intravascular excede a taxa de reenchimento desse espaço, principalmente se a resistência periférica não consegue compensar a perda do volume intravascular. Assim, enquanto a resistência periférica total aumenta durante a UF isolada, esta resposta compensatória é atenuada quando o processo de difusão está associado. Dessa forma, durante uma ultrafiltração combinada com difusão, situação em que a vasoconstrição não é evidente, a capacidade de remover volume durante a hemodiálise é primariamente dependente da capacidade de reenchimento do espaço intravascular, porque a vasoconstrição não ocorre de maneira eficaz ${ }^{(11)}$.

O ganho de peso interdialitico muito grande não é fácil de ser removido durante a hemodiálise convencional, mesmo na presença de sobrecarga de volume intravascular, pois o reenchimento deste espaço é tempo dependente. Em geral, a hipotensão arterial surge quando a taxa de UF excede 1,5 litros por hora, e a hipotensão é geralmente uma função exponencial da taxa de fluido removido (11).

Outra causa da hipotensão está relacionada a fatores cardíacos, por um débito cardíaco excessivamente dependente de uma disfunção diastólica, uma incompetência em aumentar a freqüência cardiaca, seja por uso de betabloqueadores, neuropatia urêmica, envelhecimento, entre outros (11).

A hipotensão arterial também pode estar relacionada à falta de vasoconstrição. A diminuição do volume sangüineo induzido pela diálise causa um estado no qual o débito cardíaco é limitado pelo seu enchimento, que é dependente do retorno venoso. Qualquer diminuição na resistência vascular periférica ou no enchimento cardiaco pode precipitar hipotensão (20). Situações que levam à hipotensão relacionada a esse fator são a utilização de solução de diálise com acetato de sódio, que é um vasodilatador; isquemia tecidual causada pela hipotensão e que libera adenosina que tem propriedades vasodilatadoras; a neuropatia autônoma na qual a vasoconstrição arteriolar está prejudicada em resposta à depleção de volume; medicamentos anti-hipertensivos e o uso de solução dialítica na temperatura habitualmente utilizada, ou seja, $38^{\circ} \mathrm{C}$ causando dilatação venosa e arteriolar (10)

A ingestão de alimentos durante a hemodiálise pode provocar menor constrição dos vasos de resistência em certos leitos vasculares, especialmente no leito esplâncnico. Este efeito da alimentação sobre a pressão sangüinea provavelmente dura duas horas (10)

A tecnologia moderna fornece aos profissionais da unidade de hemodiálise instrumentos que analisam, com precisão, a composição do dialisato, a taxa de UF, permitindo ajustes de acordo com as necessidades individuais de cada paciente; a temperatura do dialisato, no entanto, é regra geral aceitar que seja 
igual a do interior do organismo, entre $37^{\circ} \mathrm{C}$ e $38^{\circ} \mathrm{C}$. Porém, em publicação realizada em 1995, ela deveria ser ajustada de acordo com a temperatura do sangue nas veias do braço da fistula arteriovenosa, e não com a temperatura do interior do corpo (21)

Em um ambiente com temperatura de $22^{\circ} \mathrm{C}$ (mais ou menos a de uma unidade de diálise), um indivíduo normal tem $35,5^{\circ} \mathrm{C}$ no sangue da artéria radial, $36,5^{\circ} \mathrm{C}$ na artéria braquial e $33,2^{\circ} \mathrm{C}$ na veia da prega cubital. Embora não se tenha dados da temperatura do sangue no braço de uma pessoa com fistula arteriovenosa, é possivel admitir que o sangue que retorna do circuito extracorpóreo seja menor do que aquele da artéria radial e pouco maior do que na veia da prega cubital (21).

E sabido que variações térmicas provocam grandes efeitos sobre a função cardiovascular; assim, individuos normais expostos a um ambiente com temperatura de $40^{\circ} \mathrm{C}$ reagem com aumento no débito cardiaco chegando a 15 litros/minuto, sendo que o normal é de 3 a 7 litros/ minuto, e aumentam em sete vezes o fluxo sanguíneo cutâneo, que é o instrumento para dissipar calor do organismo. Mesmo em variações moderadas de temperatura como as que elevam a do organismo de $36,7^{\circ} \mathrm{C}$ para $37,7^{\circ} \mathrm{C}$ (variação semelhante encontrada em muitos pacientes em hemodiálise), importantes alterações hemodinâmicas também ocorrem, como a duplicação do débito cardíaco e a diminuição pela metade da resistência vascular periférica. Ao contrário, a exposição a um ambiente frio (16ㅇ), produz vasoconstrição periférica com o dobro da resistência vascular periférica, mas pouca variação no débito cardíaco(21-22)

A temperatura do dialisato afeta não somente o sangue que retorna da circulação extracorpórea, mas também da temperatura interna $(21,23)$

O efeito benéfico do dialisato frio é sugerido pela vasoconstrição e aumento da resistência periférica total através da produção aumentada de catecolaminas, redução na produção de interleucinas, fator de necrose tumoral e possivelmente do óxido nítrico, observado durante o resfriamento do corpo (23.24).

Mesmo que o dialisato a $35^{\circ} \mathrm{C}$ possa causar desconforto por levar a uma excessiva vasoconstrição e isquemia, principalmente em pacientes com circulação vascular periférica debilitada, a maioria deles tolera bem este procedimento com diminuição na incidência da hipotensão, sem interferir com a qualidade de diálise oferecida $(11,12,22,25)$

Embora as modificações ocorridas durante a hemodiálise sejam muito complexas e com vários fatores atuantes, estes dados sugerem que as condições térmicas em que o tratamento é executado podem melhorar o equilíbrio hemodinâmico do organismo e assim tornar o tratamento mais tolerável para o paciente ${ }^{(20-21)}$

\section{OBJETIVO}

Estabelecer a influência da temperatura do dialisato na ocorrência da hipotensão arterial durante o tratamento hemodialítico e avaliar o comportamento da pressão arterial quando o indivíduo é exposto a um tratamento hemodialítico com dialisato a $35^{\circ} \mathrm{C}$ e a $372 \mathrm{C}$.

\section{CASUÍSTICA E MÉTODOS}

O estudo consta de 21 pacientes, selecionados entre aqueles em hemodiálise crônica com história de episódios de hipotensão arterial sintomática durante tratamento de quatro horas.

Os pacientes estudados possuíam média de idade de 46,61 anos \pm 13,98, mediana de 50 anos, variando de 27 a 69 anos; $07(33,33 \%)$ do sexo masculino e 14 $(66,67 \%)$ do sexo feminino; $16(76,20 \%)$ eram brancos e $05(23,80 \%)$ eram negros. O tempo médio em regime de hemodiálise era de 69,09 meses \pm 57 , 80, a mediana de 68 meses, variando de 02 a 280 meses.

As etiologias das doenças básicas da IRC foram glomerulonefrite crônica em nove pacientes $(42,85 \%)$, nefroesclerose hipertensiva em oito pacientes $(38,09 \%)$, nefropatia diabética em dois pacientes $(9,52 \%)$, pielonefrite crônica em um paciente $(4,76 \%)$ e causa desconhecida em um paciente $(4,76 \%)$.

Hipotensão arterial sintomática foi definida como pressão arterial sistólica abaixo de $90 \mathrm{mmHg}$ ou queda em 25\% desse valor quando a pressão sistólica basal do paciente fosse de 90-100 $\mathrm{mmHg}$, associada a sintomas como zumbido, bocejos, fraqueza muscular, náuseas e vômitos, cãibras, sudorese, taquicardia, dor precordial e confusão mental e que necessitavam alguma forma de intervenção, como infusão endovenosa rápida de solução fisiológica a 0,9\%, sódio hipertônico (NaCI 20\%), interrupção da taxa de UF, diminuição da velocidade do fluxo da bomba de sangue ou associação dessas medidas.

Para avaliação do efeito do dialisato frio sobre a pressão arterial, foi realizado estudo prospectivo, de um grupo experimental.

Cada paciente envolvido no estudo foi avaliado por quatro sessões de hemodiálise consecutivas, variando a temperatura do dialisato, sendo as duas primeiras a $35^{\circ} \mathrm{C}$ e as outras a $37^{2} \mathrm{C}$. As sessões de hemodiálise foram realizadas em máquinas individuais de proporção com dispositivo próprio para controle da temperatura (Baxter 1550, Tampa, EUA, 1992/93).

O filtro de diálise utilizado foi do tipo capilar de polissulfona de $1,3 \mathrm{~m}^{2}$, com coeficiente de ultrafiltração de $5,5 \mathrm{ml} / \mathrm{hr} / \mathrm{mmHg}$ (Hemoflow $\mathrm{F}_{6}$, Fresenius, Alemanha). As sessões foram realizadas três vezes por semana com duração de quatro horas. A solução do dialisato era composta de $\mathrm{Na}=140,0 \mathrm{mEq} / 1, \mathrm{~K}=2,0 \mathrm{mEq} /$ 1, $\mathrm{Ca}=3,5 \mathrm{mEq} / 1, \mathrm{Mg}=0,8 \mathrm{mEq} / 1, \mathrm{Cl}=110 \mathrm{mEq} / 1$, Bicarbonato $=35,0 \mathrm{mEq} / 1$ e $\mathrm{pH}=7,2$; o fluxo de sangue foi mantido em $350 \mathrm{ml} / \mathrm{min}$ e de dialisato em $500 \mathrm{ml} / \mathrm{min}$.

Os pacientes realizaram o tratamento sentados em poltronas reclináveis em uma sala com temperatura ambiente que variou entre $23^{2} \mathrm{C}$ a $25^{\circ} \mathrm{C}$.

A pressão arterial foi verificada pelo método convencional de ausculta, utilizando esfigmoma- 
nômetro de coluna de mercúrio, Tycos, realizada pela equipe de enfermagem da unidade de diálise, sendo padronizado a primeira fase de Korotkoff para pressão sistólica e a quinta fase para pressão diastólica; as medidas, em duplicata, foram realizadas 15 minutos antes de iniciar a sessão, na primeira, segunda, terceira e quarta horas de tratamento e 15 minutos após terminado a terapia, e considerou-se a média como parâmetro para as análises estatísticas.

Os medicamentos anti-hipertensivos utilizados por 13 pacientes $(61,90 \%)$ foram mantidos, e nos dias de tratamento hemodialítico eram administrados após a sessão.

A ultrafiltração era programada para atingir o "peso seco" de cada paciente, sendo que o peso pré-hemodiálise e pós-hemodiálise foram verificados em uma mesma balança eletrônica.

\section{ANÁLISE ESTATÍSTICA}

Para avaliar o efeito das temperaturas de $35^{\circ} \mathrm{C}$ e $37{ }^{2} \mathrm{C}$ sobre as pressões arteriais sistólica e diastólica dos pacientes utilizou-se a estatística descritiva e a análise de variância para medidas repetidas, ao nível de significância de $5 \%$.

\section{RESULTADOS}

As médias e os desvios padrões referentes às aferições das pressões arteriais sistólica e diastólica (em $\mathrm{mmHg}$ ), durante duas sessões de hemodiálise a $35^{2} \mathrm{C}$ e duas a $37^{\circ} \mathrm{C}$, dos 21 pacientes avaliados no estudo, nos periodos de 15 minutos pré-diálise, $1^{\mathrm{a}}, 2^{\mathrm{a}}$, $3^{\mathrm{a}}, 4^{\mathrm{a}}$ hora de tratamento e 15 minutos após o término da terapia dialítica são mostradas na tabela 1 .

As pressões arteriais sistólica e diastólica nas sessões de hemodiálise a $35^{\circ} \mathrm{C}$ e a $37{ }^{2} \mathrm{C}$ mostraram diferenças estatisticamente significativas com $\mathrm{p}=0,0133$ e $\mathrm{p}=0,0045$, respectivamente, quando comparadas aos valores iniciais, pré-diálise, mas não se verificou influência pressórica diferente quantitativamente nesse estudo, com dialisato em temperatura baixa.

Tabela 1- Resultados referentes às pressões arteriais sistólica e diastólica dos pacientes em hemodiálise com temperaturas de dialisato a $37^{\circ} \mathrm{C}$ e $35^{\circ} \mathrm{C}$, em seis avaliações. Londrina, PR., 1999.

\begin{tabular}{|c|c|c|c|c|c|c|c|c|}
\hline \multirow[t]{2}{*}{ Avaliações } & & Pressão & Sistólica & & \multicolumn{2}{|c|}{ Pressão } & Diastólica & \\
\hline & \multicolumn{2}{|c|}{$37^{\circ} \mathrm{C}$} & \multicolumn{2}{|c|}{$35^{\circ} \mathrm{C}$} & \multicolumn{2}{|c|}{$37^{\circ} \mathrm{C}$} & \multicolumn{2}{|c|}{$35^{\circ} \mathrm{C}$} \\
\hline Pré & 133,4 & $23,2^{a *}$ & 143,0 & 21,4 & 84,2 & $10,9^{*}$ & 90,0 & 12,9 \\
\hline $1 \mathrm{~h}$ & 125,9 & 19,9 & 136,6 & 24,9 & 82,6 & 11,8 & 86,4 & 15,7 \\
\hline $2 \mathrm{~h}$ & 120,6 & 21,5 & 130,5 & 21,5 & 79,5 & 13,3 & 82,5 & 12,9 \\
\hline $3 \mathrm{~h}$ & 115,0 & 24,6 & 119,3 & 22,8 & 75,1 & 13,2 & 77,1 & 12,7 \\
\hline $4 \mathrm{~h}$ & 113,6 & 27,8 & 115,9 & 25,0 & 72,1 & 12,0 & 77,4 & 13,9 \\
\hline Pós & 120,7 & 27,2 & 123,3 & 21,4 & 76,8 & 11,2 & 82,6 & 12,0 \\
\hline
\end{tabular}

Valores expressos como média \pm desvio padrão $\left(\mathrm{X}_{\mathrm{f}} \mathrm{DP}\right)$

* Temperatura de $37^{\circ} \mathrm{C}$ difere de $35^{\circ} \mathrm{C}$ com $\mathrm{p}<0,05$
As variações entre as medidas periódicas das pressões arteriais sistólica e diastólica, também foram estatisticamente significativas com $\mathrm{p}<0,001$.

\section{PRESSÃO SISTÓLICA MEDIA}

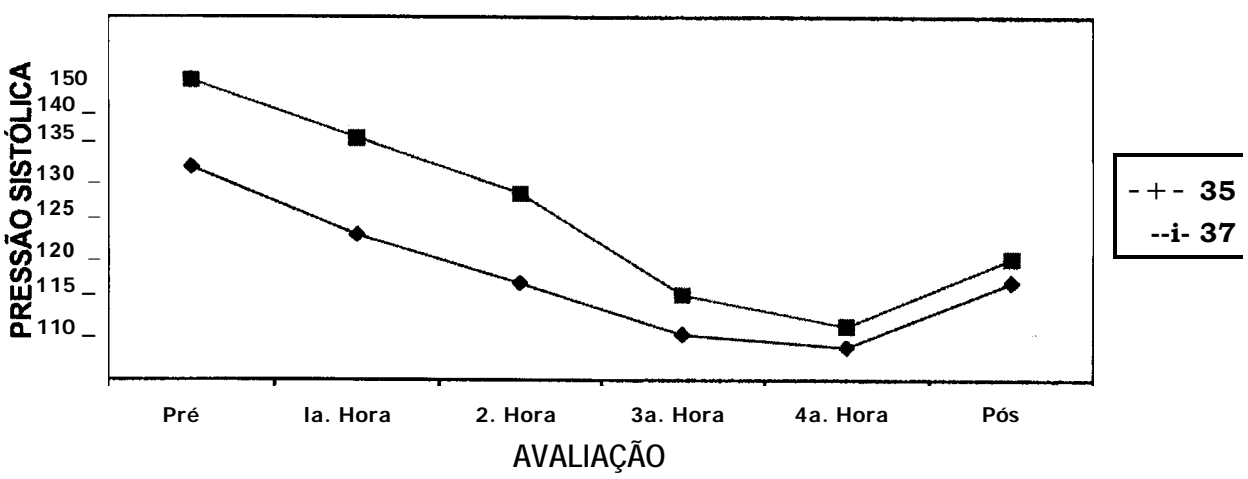

Figura 1 - Pressão arterial sistólica média dos pacientes em hemodiálise com temperaturas de dialisato a $35^{\circ} \mathrm{C}$ e $37^{\circ} \mathrm{C}$, em seis avaliações. 


\section{PRESSÃO DIASTÓLICA MEDIA}

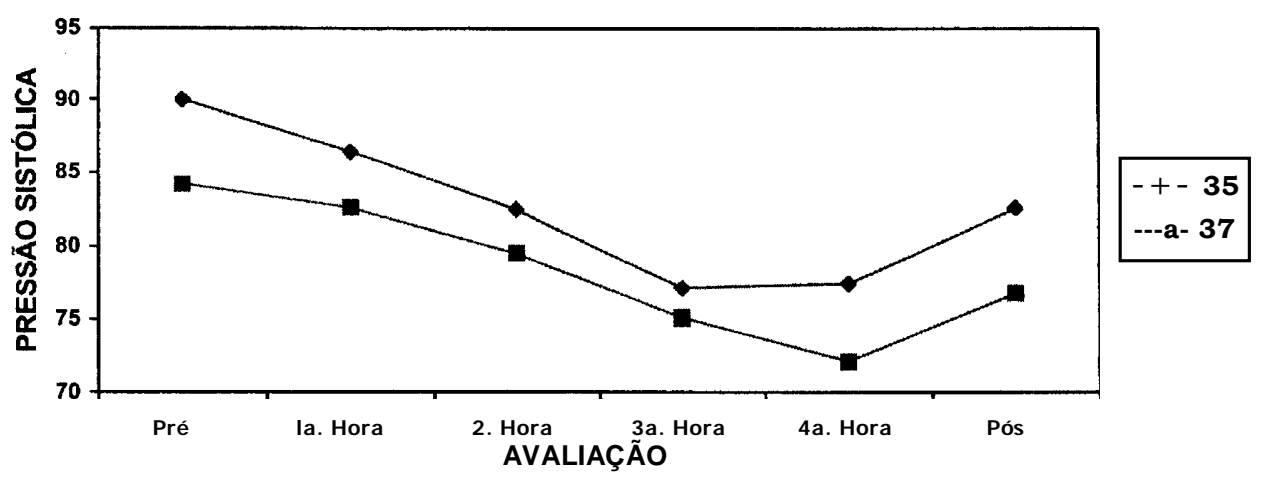

Figura 2 - Pressão arterial diastólica média dos pacientes em hemodiálise com temperaturas de dialisato a $35^{\circ} \mathrm{C}$ e $37^{\circ} \mathrm{C}$, em seis avaliações.

A Tabela 2 mostra os valores das reduções das pressões arteriais sistólica e diastólica na $1^{\mathrm{a}}, 2^{\mathrm{a}}, 3^{\mathrm{a}}$, $4^{a}$ horas de tratamento e pós-diálise em relação à pressão arterial pré-diálise, para cada uma das temperaturas estudadas. Com exceção da pressão arterial diastólica da $1^{a}$ hora $(p>0,13)$ os resultados apresentaram diferenças significativas e crescentes no decurso do procedimento com $\mathrm{p}<0,05$.

Tabela 2 - Resultados referentes à redução das pressões arteriais sistólica e diastólica dos pacientes em hemodiálise com temperaturas de $37^{\circ} \mathrm{C}$ e $35^{\circ} \mathrm{C}$, em relação à pré-avaliação. Londrina, PR., 1999. ${ }^{a}$

\begin{tabular}{l|c|cc|cc|cc}
\hline \multirow{2}{*}{ Avaliações } & \multicolumn{2}{|c|}{ Pressão } & \multicolumn{2}{c|}{ Sistólica } & \multicolumn{2}{c}{ Pressão } & \multicolumn{2}{c}{ Diastólica } \\
\cline { 2 - 8 } & $37^{\circ} \mathrm{C}$ & \multicolumn{2}{|c|}{$35^{\circ} \mathrm{C}$} & \multicolumn{2}{c}{$37^{\circ} \mathrm{C}$} & \multicolumn{2}{c}{$35^{\circ} \mathrm{C}$} \\
\hline $1 \mathrm{~h}$ & $7,513,4 a^{*}$ & 6,4 & 12,6 & 1,5 & 7,6 & 3,6 & 10,6 \\
\hline $2 \mathrm{~h}$ & $12,817,2^{*}$ & 12,5 & 14,8 & 4,6 & $10,1^{*}$ & 7,5 & 11,4 \\
\hline $3 \mathrm{~h}$ & $18,419,5^{*}$ & 23,7 & 19,5 & 9,0 & $10,2^{*}$ & 12,8 & 13,4 \\
\hline \multirow{2}{*}{$4 \mathrm{~h}$} & $19,922,1^{*}$ & 27,0 & 22,1 & 12,0 & $10,6^{*}$ & 12,6 & 14,9 \\
\hline Pós & $10,122,5^{*}$ & 19,6 & 17,9 & 1,5 & $10,2^{*}$ & 7,4 & 11,2 \\
\hline
\end{tabular}

Valores expressos como média \pm desvio padrão (X $\pm \mathrm{DP})$ *

Temperatura de $37^{\circ} \mathrm{C}$ difere de $35^{\circ} \mathrm{C}$ com $\mathrm{p}<0,05$

Durante o estudo foi observado que das 42 sessões de hemodiálise com temperatura a $35^{\circ} \mathrm{C}$, ocorreram 18 episódios (9 pacientes) de hipotensão arterial sintomática, necessitando tratamento através de infusão de solução salina a $0,9 \%$, sendo que dois episódios ocorreram na $2^{a}$ hora, sete, na $3^{\text {a }}$ hora e nove, na $4^{\text {a }}$ hora. Nas outras 42 sessões com temperatura a $37^{\circ} \mathrm{C}$, ocorreram 21 episódios (12 pacientes) de hipotensão arterial, sendo três, na $2^{a}$ hora, oito, na $3^{\text {a }}$ hora e dez, na $4^{a}$ hora. As principais sintomatologias observadas pela equipe de enfermagem e relatadas pelos pacientes nos episódios de hipotensão, foram sudorese, bocejos, cefaléia e fraqueza muscular.

\section{DISCUSSÃO}

A hipotensão arterial é a principal complicação aguda que ocorre durante as sessões de hemodiálise e várias medidas têm sido utilizadas no intuito de tornar esse procedimento mais confortável e seguro para o paciente; isso inclui o aumento na concentração de sódio do dialisato, o uso de bicarbonato de sódio como solução tampão e de membranas biocompativeis e a diminuição da temperatura do líquido de diálise.

Como mostra a tabela 1 e as figuras 1 e 2, ocorreu comportamento semelhante com queda gradativa nas pressões arteriais sistólica e diastólica em ambas as 
temperaturas estudadas $\left(35^{\circ} \mathrm{C}\right.$ e $\left.37^{\circ} \mathrm{C}\right)$ com resultado estatisticamente significativo. A curva das pressões arteriais na temperatura a $37^{\circ} \mathrm{C}$ permaneceu constantemente em um nivel inferior da observada a $35^{\circ} \mathrm{C}$, porém, como os valores das pressões pré-diálise já eram diferentes e superiores com dialisato a $35^{\circ} \mathrm{C}$, não podemos afirmar que essa permanência em limites inferiores a $37^{\circ} \mathrm{C}$ tenha sido influenciada pela temperatura.

Em pesquisa realizada em 1993 foi avaliada a pressão arterial média de 12 pacientes do sexo masculino divididos em dois grupos, um formado de normotensos ou hipotensos pré-diálise (pressão arterial média $<90$ $\mathrm{mmHg}$ ) e outro de ganhadores de peso (> $4 \mathrm{Kg}$ ) no período interdialítico, relacionadas em duas sessões de hemodiálise a $35^{\circ} \mathrm{C}$ e a $37^{\circ} \mathrm{C}$, com intervalo de uma semana entre cada avaliação. O resultado encontrado foi uma diferença significativa entre as pressões arteriais médias a $35^{\circ} \mathrm{C} \mathrm{e} 37^{\circ} \mathrm{C}$, e menores com o uso de dialisato a $37^{\circ} \mathrm{C}$ no decorrer dos procedimentos. Ainda neste estudo, no período pré-dialítico, a pressão arterial média era maior a $35^{\circ} \mathrm{C}$ do que a $37^{\circ} \mathrm{C}$, porém, essa diferença não era estatisticamente significativa (12).

Em nosso estudo verificamos a ocorrência de episódios de hipotensão sintomática nas duas temperaturas avaliadas, $\left(53,80 \%\right.$ a $37^{\circ} \mathrm{C}$ e $46,20 \%$ a $\left.35^{\circ} \mathrm{C}\right)$, sendo relativamente menor $(7,69 \%)$ nas sessões de hemodiálise com dialisato frio.

Outro estudo acompanhou oito pacientes por um periodo de 12 meses realizando hemodiálise por seis meses a $35^{\circ} \mathrm{C}$ e seis meses a $37^{\circ} \mathrm{C}$ e observou-se que, embora o uso do dialisato frio não tenha eliminado a hipotensão induzida pela diálise houve redução em cerca de $13,5 \%$ na sua incidência $\left(47,4 \%\right.$ a $37^{\circ} \mathrm{C}$ e $33,9 \%$ a $\left.35^{\circ} \mathrm{C}\right)(11)$

Em dois hospitais no Canadá foram avaliadas 10 sessões de hemodiálise a $35^{\circ} \mathrm{C}$ e a $37^{\circ} \mathrm{C}$ em 128 pacientes e observou-se episódios de hipotensão arterial sintomática em ambas, com incidência de 5,5\% a $35^{\circ} \mathrm{C}$ e $11,2 \%$ a $37^{\circ} \mathrm{C}$, diferença de $5,7 \%$ (23),

$\mathrm{Na}$ literatura ainda encontramos estudo que constatou 18 episódios de hipotensão arterial sintomática, necessitando de tratamento, durante hemodiálise a $37^{\circ} \mathrm{C}$, contra nenhuma ocorrência em hemodiálise a $35^{\circ} \mathrm{C}(12)$

Observamos que as hipotensões sintomáticas que necessitaram tratamento com infusão de solução salina a $0,9 \%$, foram registradas com maior freqüência $(87,17 \%)$ nas 3ạs e 4as da terapia dialítica, estando em concordância com outras publicações $(10,14,18)$

A maioria, cerca de $90 \%$ dos nossos pacientes, queixaram-se de desconforto pela intensa sensação de frio durante o tratamento com diálise a $35^{\circ} \mathrm{C}$, porém, não houve qualquer outra intercorrência, nem a necessidade de interrupção do tratamento.

A sensação de frio também foi relatada por pacientes em outros estudos que utilizaram essa medida terapêutica (23-26), porém, contrário a esses relatos, um estudo concluiu que o procedimento a $35^{\circ} \mathrm{C}$ foi bem tolerado, embora tenha aumentado o número de cãibras musculares ${ }^{(11)}$.

Em pesquisa realizada para relacionar a temperatura corporal na ocorrência de hipotensão sintomática durante sessões de hemodiálise concluiu que, somente os pacientes com temperatura corporal pré-diálise menor que $36^{\circ} \mathrm{C}$ seriam elegiveis para o tratamento com dialisato frio, no intuito de se obter melhora na estabilidade hemodinâmica (23).

\section{CONCLUSÃo}

Houve diferença estatisticamente significativa entre as pressões sistólica e diastólica nas duas temperaturas estudadas, porém, não podemos concluir que houve influência da temperatura do dialisato no comportamento das pressões arteriais, já que elas eram diferentes no periodo pré-diálise em cada temperatura estudada.

Observamos queda progressiva das pressões arteriais sistólica e diastólica no decorrer das sessões de hemodiálise, com temperatura do dialisato a $35^{\circ} \mathrm{C}$ e $37^{\circ} \mathrm{C}$, com diferença estatisticamente significativa. Ocorreram episódios de hipotensão arterial sintomática em ambas as temperaturas estudadas $\left(35^{\circ} \mathrm{C}\right.$ e $\left.37^{\circ} \mathrm{C}\right)$, com incidência relativamente menor $(7,69 \%)$ a $35^{\circ} \mathrm{C}$. A maior incidência $(87,17 \%)$ dos episódios de hipotensão arterial sintomáticos surgiu nas $3^{\text {as }} 4^{\text {as }}$ oras do tratamento hemodialítico.

$\mathrm{O}$ método de hemodiálise a frio $\left(35^{\circ} \mathrm{C}\right)$ não mostrou em nosso estudo, resultados diferentes da temperatura habitualmente utilizada $\left(37^{\circ} \mathrm{C}\right)$.

\section{REFERÊNCIAS BIBLIOGRÁFICAS}

(1) Cordova HR, Penab JE, Maldonado MM. Clinical manifestations and complications of the uremic state. In: Jacobson HR, Striker GE, Klahr S. The principles and practice of nephrology. Philadelphia: B.C. Decker;1991. cap.118, p.690-698.

(2) Eknoyan G. Treatment of the uremic syndrome and its complications. In: Jacobson HR, Striker GE, Klahr S. The principles and practice of nephrology. Philadelphia: B. C. Decker;1991. cap. 119, p. 699-705.

(3) D'avila DO, Figueiredo AE. Métodos de depuração extrarenal: hemodiálise, diálise peritoneal e novas técnicas. In: Riella MC. Princípios de nefrologia e distúrbios hidroeletroliticos. 3nded. Rio de Janeiro: Guanabara Koogan; 1996. cap.48, p.607-614.

(4) Sorkin ML, Diaz-Buxo JA. Fisiologia da diálise peritoneal. In: Daugirdas JT, Ing TS. Manual de diálise. $2^{\text {nd }}$ ed. Rio de Janeiro: Medsi; 1996. cap.13, p.227-241. 
(5) Keshaviah P. Technology and clinical application of hemodialysis. In: Jacobson HR, Striker GE, Klahr S. The principles and practice of nephrology. Philadelphia: B.C.Decker; 1991. cap. 125, p.740-749.

(6) MassolaVC. Métodos dialiticos. In: Cruz J, Praxedes JN, Cruz HMM. Nefrologia, São Paulo: Sarvier; 1995. cap.18, p.201-218.

(7) Van Stone JC, Daugirdas JT. Princípios fisiológicos. In: Daugirdas JT, Ing TS. Manual de diálise. 2 nd ed. Rio de Janeiro: Medsi; 1996. cap.2, p. 13-27.

(8) Schulman G. Complications of hemodialysis. In: Jacobson HR, Striker GE, Klahr S. The principles and practice of nephrology. Philadelphia: B-C.Decker; 1991. cap.127, p.757765-

(9) Riella MC. Princípios de nefrologia e distúrbios hidroeletroliticos. 3.ed. Rio de Janeiro: Guanabara Koogan, 1996. cap. 36 , p. $456-476$

(10) Bregman H, Daugirdas JT, Ing TS. Complicações durante a hemodiálise. In: Daugirdas JT, Ing TS. Manual de diálise. 2n ${ }^{d}$ ed. Rio de Janeiro: Medsi; 1996. p.139-154.

(11) Marcén R, Quereda C, Orofino L, Lamas S, Teruel JL, Matesanz R, Ortuno J. Hemodialysis with lowtemperature dialysate: a long-term experience. Nephron 1988; 49(1):2932.

(12) Jost CM, Agarwal R, Khair-el-Din T, Grayburn PA, Victor RG, Henrich WL. Effects of cooler temperature dialysate on hemodynamic stability in "problem" dialysis patients. Kidney Int 1993; 44(3):606-612.

(13) Friess U, Rascher W, Ritz E, Gross R Failure of argininevasopressin and other pressor hormones to increase in severe recurrent dialysis hypotension. Nephrol Dial Transplant 1995; 10(8):1421-1427.

(14) Leunissen KM, Kooman JP, Van Kuij KW, Van der Sande F, Luck AJ, Van Hooff JR Preventing haemodynamic instability in patients at risk for intradialytic hypotension. Nephrol Dial Transplant 1996; 11 (Suppl 2):11-15.

(15) Zoccali C, Tripepi G, Mallamaci F, Panuccio V. The heart rate response pattern to dialysis hypotension in haemodialysis patients. Nephrol Dial Transplant 1997; 12(3):519-523.

(16) Bazzato G, Coli U, Landini S, Lucatello S, Fracasso A, Morachiello P. et al. Temperature monitoring in dialysisinduced hypotension. Kidney Int 1985; 17 (Suppl): s161-165-

(17) Nakamoto M. The mechanism of intradialytic hypotension in diabetic patients. Nippon Jinzo Gakkai Shi 1994; 36(4): 374-381.

(18) Lin SH, Chu P, Yu FC, Diang LK, Lin YE Increased nitric oxide production in hypotensive hemodialysis patients. Asaio $J$ 1996; 42(5):895-899.

(19) Levy FL, Grayburn PA, Foulks CJ, Brickner ME, Henrich WL. Improved left ventricular contractility with cool temperature hemodialysis. Kidney Int 1992; 41(4):961-965.
(20) Schneditz D, Martin K, Kramer M, Kenner T, Skrabal E. Effect of controlled extracorporeal blood cooling on ultrafiltration-induced blood volume changes during hemodialysis. J Am Soc Nephrol 1997; 8(6):956-964.

(21) Maggiore Q, Dattolo P, ,.Piacenti M, Morales MA, Pelosi G, Pizzarelli F, Cerrai T. Thermal balance and dialysis hypotension. Int J Artif Organs 1995; 18(9):518-25.

(22) Yu AW, Ing TS, Zabaneh RI, Daugeridas JT. Effect of dialysate temperature on central hemodynamics and urea kinetics. Kidney Int 1995; 48(1):237-243.

(23) Fine A, Penner B. The protective effect of cool dialysate is dependent on patients' predialysis temperature. Am J Kidney Dis 1996; 28(2): 262-265.

(24) Costantino ML, Cairo G, Fumero R. Haemodynamic alteration in patients undergoing chronic haemodialysis. Int J Artif Organs 1997; 20(11): 610-613.

(25) Provenzano R, Sawaya B, Frinak S, Polaschegg HD, Roy T, Zasuwa G. et al. The effect of cooled dialysate on thermal energy balance in hemodialysis patients. Asaio Trans 1988; 34(3): p.515-518.

(26) Kerr PG, Van Bakel C, Dawborn JK. Assessment of the symptomatic benefit of cool dialysate. Nephron 1989; 52(2): 166-169.

\section{Artigo recebido em 28101100}

Artigo aprovado em 24/07102 